\title{
Cloud Screening on Monochromatic Images of MSU-100M (Meteor-M №2) Based on Spatial Analysis and Ground Objects Boundaries
}

\author{
Pavel A. Kolbudaev, Dmitry E. Plotnikov, Sergey A. Bartalev \\ Space Research Institute, Russian Academy of Sciences, Moscow, Russia \\ kolbudaev@d902.iki.rssi.ru
}

\begin{abstract}
The study is devoted to the development of a method for clouds detection on monochromatic images of the MSU100M instrument onboard Meteor-M №2 satellite. Being preparatory for cloud masking, a method for recalibrating of input data and a new format for image spatial arrangement and processing were implemented and described. MSU-100M images localized registration was based on MODIS composite images, following further enhancement based on the comparison with the objects boundaries derived from the segmentation of OLI cloudfree imagery. As an example, we used near-infrared bands data derived from OLI (Landsat-8) and MODIS (Terra) devices. The specific of the methodology is that it requires data from one MSU-100M spectral channel only.
\end{abstract}

Keywords: remote sensing, Meteor-M series, MSU-100M, MODIS, Landsat, OLI, cloud detection 


\title{
МЕТОД ВЫЯВЛЕНИЯ ОБЛАЧНОСТИ ПО ДАННЫМ ПРИБОРА МСУ- 100M (МЕТЕОР-M №2) НА ОСНОВЕ ПРОСТРАНСТВЕННОГО АНАЛИЗА МОНОХРОМНЫХ ИЗОБРАЖЕНИЙ И ГРАНИЦ ОБЪЕКТОВ ЗЕМНОЙ ПОВЕРХНОСТИ
}

\author{
П.А. Колбудаев, Д.Е. Плотников, С.А. Барталев \\ Институт космических исследований РАН, Москва, Россия \\ kolbudaev@d902.iki.rssi.ru
}

\begin{abstract}
Данная работа посвящена разработке метода выявления облачности на монохромных изображениях, получаемых прибором МСУ-100М со спутника Метеор-М №2. Для решения задачи выделения облачности разработан метод докалибровки поступающих данных и применен новый формат для пространственной организации и обработки спутниковых изображений. Также был разработан метод локализованного уточнения географической привязки спутниковых изображений МСУ-100М на основе композитных изображений MODIS и дальнейшего улучшения привязки с использованием границ предварительно выделенных сегментов, полученных по данным прибора OLI (Landsat-8). В качестве демонстрации в работе использовались измерения приборов OLI (Landsat-8) и MODIS (Terra), полученные в ближнем инфракрасном диапазоне длин волн. Особенность разработанного алгоритма состоит в возможности его применения для выявления облачности на основе данных спутниковой съемки только в соответствующем спектральном канале.
\end{abstract}

Ключевые слова: дистанционное зондирование, Метеор-М, МСУ-100M, MODIS, Landsat, OLI, детектирование облачности

\section{Введение}

Прибор МСУ-100М осуществляет съемку земной поверхности со спутника Метеор-М №2, выведенного на орбиту 8 июля 2014 года. Параметры орбиты спутника и ширина полосы обзора двух камер прибора МСУ-100М позволяют достичь достаточно высокой периодичности наблюдений, составляющей около трех суток на широте средней полосы России. Достигаемая при этом частота спутниковых наблюдений указанным прибором в сочетании с уровнем пространственного разрешения получаемых данных (около 60 метров) приводит к достаточно востребованному сочетанию характеристик «частота-разрешение», несколько опережая по этому параметру хорошо зарекомендовавший себя прибор AWiFS (ResourceSat-2A). MCУ-100M способен регистрировать электромагнитное излучение в зеленом (0,535-0,575 мкм), красном (0,63-0,68 мкм) и ближнем ИК (0,76-0,90 мкм) диапазонах длин волн. Однако оптическая схема прибора делает невозможным одновременную регистрацию данных во всех спектральных каналах, приводя при этом к необходимости разработки специальных методов предварительной обработки получаемых изображений, учитывающих их геометрические особенности, вызванные фактором временной рассинхронизации и пространственного параллакса многоспектральных измерений. 
Учитывая наличие свободного доступа к данным МСУ-100М и удобное сочетание характеристик прибора по величинам пространственного разрешения и частоты наблюдений, получаемые спутниковые изображения представляют значительный интерес для оценки характеристик земной поверхности, картографировании и оценки состояния сельскохозяйственной и лесной растительности, а также решения других задач. Однако до настоящего времени возможности тематической интерпретации данных МСУ-100М в подобного рода приложениях были в значительной степени ограничены сложностью эффективного решения задач предварительной обработки изображений, способной, в частности, обеспечить их точную географическую привязку и очистку от влияния облачности.

В ИКИ РАН ведется оценка возможностей практического использования спутниковых данных МСУ-100М на основе автоматических технологий, что позволило разработать ряд новых подходов к проведению предварительной обработки спутниковых изображений, позволяющих обеспечить достаточно эффективное решение указанных проблем, сопряженных с конструктивными особенностями съемочной системы. Разработанные подходы в автоматическом режиме позволяют осуществлять следующие группы операций обработки данных:

- Реорганизация данных на основе гранул, определяемых границами регулярной сети градусных ячеек для повышения эффективности их дальнейшей обработки;

- Повышение точности географической привязки изображений MCУ-100M на основе использования данных, получаемых системами OLI(Landsat-8) и MODIS (Terra);

- Выявление облачности и соответствующих ей теней на изображениях MCУ-100М на основе их совместного анализа с данными OLI(Landsat-8) и MODIS (Terra).

\section{Описание алгоритма}

Конструктивные особенности прибора МСУ-100М затрудняют применение известных решений для детектирования облачности на получаемых спутниковых изображениях в силу, сопряженных с ними значительных различий геометрических условий наблюдения в зеленом, красном и БИК каналах (величина углового параллакса составляет около $9^{\circ}$ ) и возникающей в результате разницы во времени наблюдения одного и того же участка поверхности (около 20 секунд между соседними каналами). После орторектификации и пространственного совмещения разноспектральных данных на уровне земной поверхности изображения облаков остаются рассогласованными в различных спектральных каналах. В результате этого обстоятельства, а также ввиду отсутствия данных наблюдений в средней инфракрасной области спектра, использование для выделения облачности известных решений (например, метод Fmask [1]) оказывается затруднено.

На начальном этапе данные измерений МСУ-100М преобразуются в значения КСЯ на основании известных аппаратных функций для каждого спектрального канала и зенитного угла Солнца, положение которого определяется на основе астрономических формул с использованием информации о времени съемки и географических координатах местности. Пространственная структура хранения изображений МСУ-100М преобразуется к виду регулярной сети гранул (1х1 градус) в долготно-широтной картографической проекции со стандартизацией имен файлов для повышения эффективности автоматической потоковой обработки данных на больших территориях. Имена файлов включают в себя необходимую информацию о продукте, его версии, спектральном канале, регионе, дате и времени съёмки, другие необходимые характеристики данных. Для улучшения географической привязки изображений используются сегментированные 
данные Landsat в БИК спектральном диапазоне, очищенные от влияния облачности и теней с помощью усовершенствованного метода Fmask [2] и восстановленные временные ряды четырехдневных композитных изображений по данным MODIS в БИК канале.

Первоначально для уточненной географической привязки данных МСУ-100М используются данные MODIS в БИК канале. Линейное преобразование (сдвиг) сопровождается совместным анализом соответствующей пары модифицированного изображения МСУ-100М и эталонного изображения MODIS в ИК каналах приборов на близкие даты и максимизацией значения коэффициента корреляции, рассчитанных для всех пар измерений. Предварительно картографическая проекция данных MODIS приводится в соответствие с параметрами проекции изображений МСУ-100М. Вышеописанный подход применяется локально для каждого участка независимо и для каждого из них определяется величина смещения, при которой достигается максимальное значение корреляции, в дальнейшем используемое при построении маски облаков. Описанный подход позволяет обеспечить привязку данных МСУ-100 с точностью до размера пискела MODIS, что не может считаться достаточным. Следующий шаг обработки данных предполагает использование результатов сегментации изображений OLI и MCУ-100M. Сегментация основана на использовании критерия минимума расстояния в ближайшей окрестности сегмента [3]. В качестве критерия минимума расстояния используется разность среднего коэффициента отражения подстилающей поверхности для сегмента, а также величина максимального и минимального коэффициентов отражения, в целях предотвращения образования больших и слишком неоднородных сегментов. Первоначально с помощью улучшенного метода детектирования облаков и теней Fmask создается временной ряд очищенных от влияния облаков данных Landsat за вегетационный период, в результате сегментации которого вычисляется вероятность наблюдения границ сегментов в каждом пикселе с определением устойчивых границ сегментов. После сегментации и определении границ сегментов на текущем изображении МСУ-100М выполняется локализованный анализ их соответствия опорным границам по данным Landsat c расчетом метрики, характеризующей степень их совпадения. Предварительно картографическая проекция данных и пространственное разрешение OLI приводится в соответствие с параметрами изображения МСУ-100М. Вышеописанный подход также применяется локализованно. В итоге с помощью использования данных MODIS и OLI производится уточненная географическая привязка данных МСУ-100M с учетом нелинейности искажений исходного изображения. Метод выявления облачности на монохромных спутниковых изображениях, получаемых прибором МСУ-100М, основан на использовании значений их корреляции с данными MODIS, используемыми ранее на этапе уточнения географической привязки данных. Корреляция оценивалась локально и независимо для каждого фрагмента изображения размером 100 на 100 пикселей. Для фрагментов указанного размера с наличием облачности значение коэффициента корреляции оказывалось ниже, чем для безоблачных, позволяя установить порог на значение этого коэффициента для разделения классов облаков и чистой поверхности. На основе свободных от влияния облаков участков изображений определяется уравнение (прямой) соответствия между измерениями МСУ-100М и MODIS, a также отклонения от него. С помощью данного уравнения для любого пиксела изображения становится возможным определить расстояние от рассматриваемого пикселя в пространстве измерений двух приборов до соответствующей прямой, в том числе, в терминах числа дисперсий. Учитывая положение объектов класса облачности в указанном двумерном пространстве, оказывается возможным попиксельно детектировать подверженные влиянию облачности территории устанавливая пороговое значение на расстояние до прямой соответствия. Аналогичный подход позволяет детектировать и тени от облаков, характеризуемые, наоборот, отрицательным отклонением от линии соответствия. На рисунке 1 показаны результаты выделения различных по структуре облаков на изображении, полученном прибором МСУ-100М 

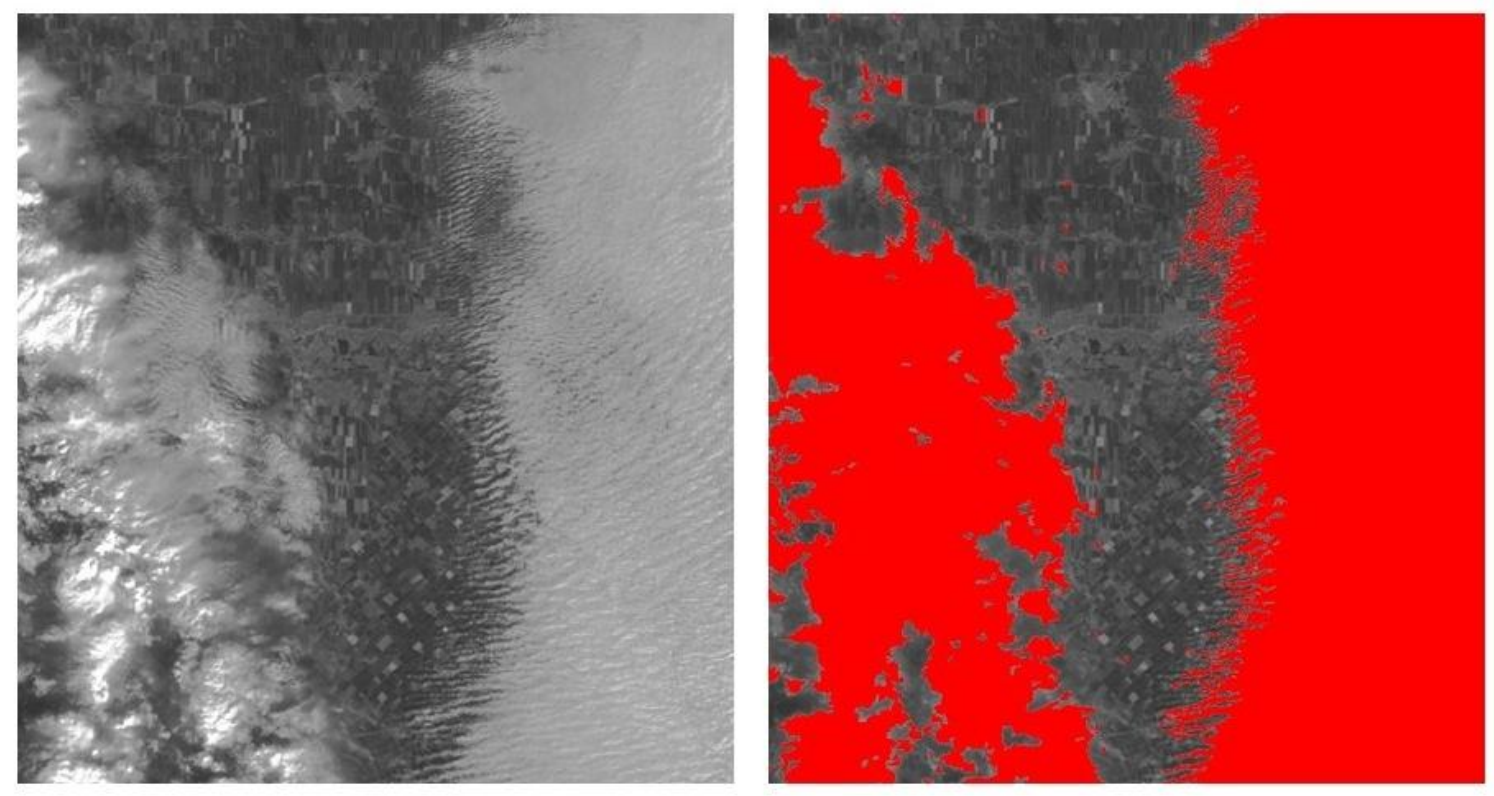

Рис 1. Фрагмент изображения, полученного прибором Метеор М2\МСУ-100М 8 октября 2016 года на территорию Ставропольского края в БИК канале (слева) и соотвествующая ему маска облачного покрова (справа, красным цветом отмечены детектированные облака)

Особенность описанного метода, основанного на пространственном анализе изображений и не требующего использования мультиканальных индексов, состоит в обеспечиваемой им возможности выявлять изображения облаков и соответствующих им теней на основе пары измерений MODIS-MCУ в одном спектральном канале.

Исследования выполнялась с использованием инфраструктуры Центра коллективного пользования системами архивации, обработки и анализа спутниковых данных ЦКП "ИКИМониторинг" для решения задач изучения и мониторинга окружающей среды [4] при финансовой поддержке государства в лице Минобрнауки России: соглашение 05.577.21.0294 «Разработка технологий автоматизированной обработки спутниковых данных дистанционного зондирования Земли для создания и поддержки информационных сервисов мониторинга лесных ресурсов и охотничьих угодий России», уникальный идентификатор проекта RFMEFI57718X0294.

\section{References}

[1] Zhe Zhu, Curtis E. Woodcock. Object-based cloud and cloud shadow detection in Landsat imagery. Remote Sensing of Environment, 118(15), 83-94

[2] Kolbudaev P.A., Bartalev S.A., Matveev A.M., Plotnikov D.E. Technology for processing time series of satellite data Landsat-TM / ETM +, Eleventh All-Russian Open Conference "Modern Problems of Remote Sensing of the Earth from Space." Moscow. IKI RAS, November 11-15, 2013. Conference Abstracts, 2013. p. 37. 
[3] Plotnikov D.E., Kolbudaev P.A., Bartalev S.A. Identification of dynamically homogeneous areas with time series segmentation of remote sensing data, Computer Optics, 2018; 42(3): 447-456. DOI: $10.18287 / 2412-6179-2018-42-3-447-456$.

[4] Loupian E.A., A.A. Proshin, M.A. Burtsev, I.V. Balashov, S.A. Bartalev, V.Yu. Efremov, A.V. Kashnitskiy, A.A. Mazurov, A.M. Matveev, O.A. Sudneva, I.G. Sychugov, V.A. Tolpin, I.A. Uvarov. IKI center for collective use of satellite data archiving, processing and analysis systems aimed at solving the problems of environmental study and monitoring, Sovremennye Problemy Distantsionnogo Zondirovaniya Zemli iz Kosmosa, 2015, 12(5), pp. 247-267. 